\title{
Aspects instationnaires de poches de cavitation : Mesures de pressions et films rapides simultanés
}

\author{
F. Joussellin, CREMHyG \\ E. Sauvage-Boutar, SEP
}

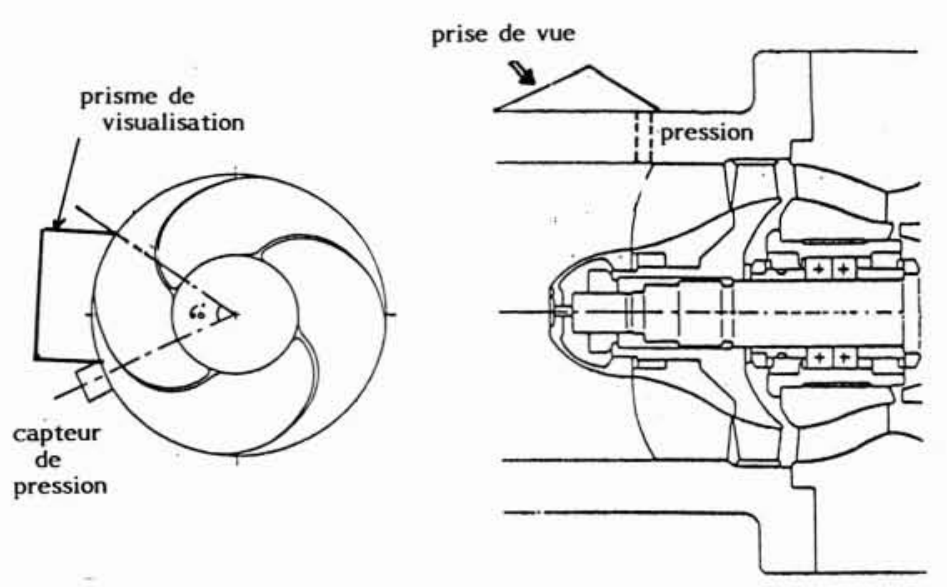

1. Schéma du montage de l'inducteur, positionnement du capteur de pression.

\section{Introduction}

La cavitation dans les pompes de moteurs-fusée limite leur capacité d'aspiration et la vitesse de rotation. Elle est aussi responsable d'instabilités de fonctionnement, de bruit et d'érosion. Cet article concerne l'étude des instabilités se développant, en particulier, sur l'inducteur de la pompe à hydrogène du moteur Vulcain (Ariane 5). Dans cet inducteur, la cavitation se développe notamment sous la forme de poches attachées sur les pales, dont les fluctuations de longueur conduisent à des charges radiales importantes, qui peuvent devenir critiques pour le fonctionnement d'une telle machine.

Afin d'étudier ces phénomènes instationnaires, des expérimentations bidimensionnelles sur veine fixe ont permis la mise en place de méthodes d'analyse, notamment à partir de signaux de pressions synchronisés à des films rapides. Puis, l'étude a été poursuivie sur l'inducteur en similitude à l'eau.

\section{Installations expérimentales}

Il s'agit de trois expériences réalisées sur des géométries en similitude, équipées de moyens de visualisation (films rapides à 7000 images/s) synchronisés à des capteurs de pression pariétale.

L'inducteur de la pompe à hydrogène du moteur Vulcain (Ariane 5) se compose d'un inducteur double grille et d'un redresseur (fig. 1). L'étude concerne principalement la première grille qui doit supporter la cavitation en fonctionnement nominal.

Un prisme en plexiglass permet de visualiser la cavitation, qui se développe sur les 4 pales de la première grille. Un capteur de pression est disposé sur le rebord du prisme, visible dans le champ de la caméra. Les paramètres de contrôle sont la vitesse de rotation $(\leqslant 8000 \mathrm{tr} / \mathrm{mn})$, le coefficient de débit et le nombre de cavitation, $\sigma$, basé sur la vitesse périphérique, Vref $=\mathrm{R} \omega$ :

$$
\sigma=(\operatorname{Pref}-\mathrm{Pv}) / 0.5 \rho \operatorname{Vref}^{2}
$$

\section{Unsteady behaviour of cavities : simultaneous pressure measurements and high speed film}

Three experiments have been made to analyse unsteadiness of cavitation phenomena on a Vulcain-type inducer: 1) water simulations on inducer, 2) hydrofoil-type and 3) venturi-type $2 D$ test-sections which modelize the suction side of the inducer blades. In the hydrofoil case, vapor clouds appear periodically. By contrast, the venturi-type test rig does not present any periodic behaviour. In the inducer, another unsteady configuration appears : a cavity pattern moves from blade to blade in the same direction as the rotation. 


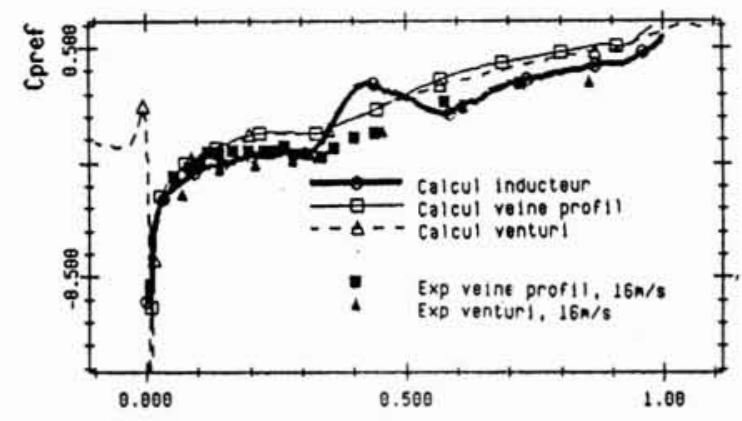

2. Répartition de pression pour les 3 expériences : inducteur (calcul S1/S2), veine "profil " et veine "Venturi " (calcul fluide parfait et expériences).

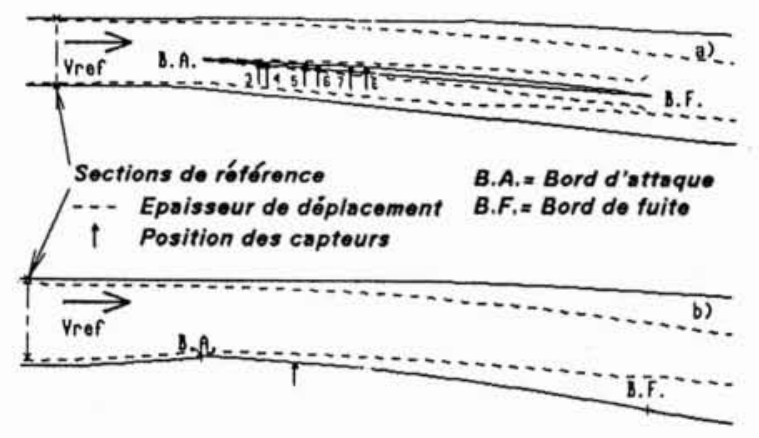

3. Géométries et couches limites (couplage calculs fluide parfait et couches limites) : a) veine "profil " b) veine "Venturi".

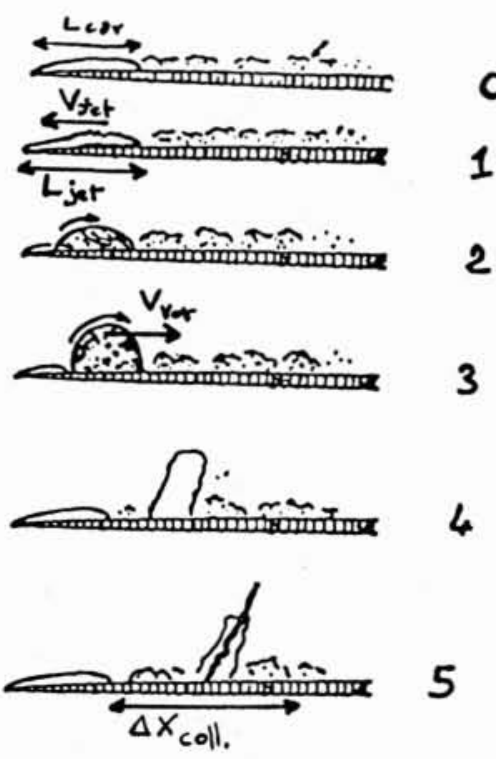

4. Scénario de lachers de structures sur une période, pour une incidence nominale et des poches courtes.
Les modèles bidimensionnels (veine de type "profil " et veine venturi) sont des géométries représentatives de l'extrados des pales de l'inducteur, réalisées avec le principal objectif d'obtenir une répartition de pression identique à celle existant sur l'extrados de l'inducteur en écoulement subcavitant. Cette distribution de pression sur l'inducteur (fig. 2) est calculée par un code S1/S2 fluide parfait, sur la surface aube à aube médiane [KUENY, DesClaux, 1989]. La comparaison des 3 distributions de pression est exprimée en terme de coefficient de pression, $\mathrm{Cp}=(\mathrm{P}-\mathrm{Pref})$ / $0.5 \rho \mathrm{Vref}^{2}$, basé sur des grandeurs de références à l'amont.

L'aubage de la veine "profil " (fig. $3 a$ ), déterminée à partir d'un calcul S1/S2 sur l'extrados d'une pale de l'inducteur, est pourvu d'une jambe qui permet de l'incliner selon l'incidence choisie ; 2 incidences ont été testées dont l'une correspond à l'incidence nominale de l'inducteur. Cette veine est équipée de 6 capteurs de pression pariétale (numérotés de 3 à 8 ). La vitesse à l'entrée varie de 9 à $32 \mathrm{~m} / \mathrm{s}$.

La veine "Venturi " (fig. $3 b$ ) est plus compacte et plus simple d'utilisation. Sa géométrie a été calculée à partir de la veine " profil " pour l'incidence nominale, en utilisant un code 2D basé sur un écoulement potentiel.

Les 2 modèles bidimensionnels ont des plages de fonctionnement identiques $(0.3<\sigma<0.7)$.

Les caractéristiques hydrauliques pour les 3 géométries sont répertoriées dans le tableau ci-dessous :

\begin{tabular}{|l|c|c|c|}
\hline & Inducteur & Veine Profil & Veine Venturi \\
\hline $\mathrm{V}_{\text {ret }}$ & $70 \mathrm{~m} / \mathrm{s}$ & $40 \mathrm{~m} / \mathrm{s}$ & $20 \mathrm{~m} / \mathrm{s}$ \\
Reynolds & $5.10^{7}$ & $3.10^{7}$ & $5.10^{6}$ \\
$\left(\mathrm{~V}_{\text {ret }} \cdot \mathrm{c} / \mathrm{v}\right)$ & 25 & 11 & 5.8 \\
$\mathrm{c} / \mathrm{h}$ & 0.17 & 0.14 & 0.11 \\
$\mathrm{~b} / \mathrm{c}$ & & & \\
\hline
\end{tabular}

$\mathrm{c}=$ corde $; \mathrm{h}=$ hauteur $; \mathrm{b}=$ largeur.

\section{Veine "profil "}

La configuration de cette veine a permis d'étudier 2 incidences pour une vitesse amont variant de $9 \mathrm{~m} / \mathrm{s}$ à $32 \mathrm{~m} / \mathrm{s}$, et des poches de longueur maximum égale à $20 \%$ de la corde.

Pour l'incidence nominale et pour des poches courtes $(\sigma$ grand), des détachements de poches périodiques ont pu être mis en évidence sur les films rapides et détaillés dans le scénario décrit à la figure 4 :

*seq. 1: un frémissement de la surface se manifeste audessus de la poche par un changement de granulométrie, simultanément un jet rentrant de liquide se propage vers l'amont sous la poche à la vitesse, $\mathrm{V}_{\text {jet }}$. 
${ }^{*}$ seq. 2 et 3 : quand le jet atteint le bord d'attaque, un vortex apparaît au début de la poche, dont l'axe est perpendiculaire à l'écoulement. Le diamètre de ce vortex augmente, tout en étant convecté vers l'aval à la vitesse, $\mathrm{V}_{\text {vor }}$. Ainsi, 2 zones distinctes apparaissent : la poche attachée et le vortex (structure diphasique détachée).

${ }^{*}$ seq. 4 et $5:$ le vortex s'élève au centre de la veine en s'affinant et prend une forme caractéristique de tourbillon en «fer à cheval», qui collapse alors qu'un autre cycle commence.

Le déroulement d'un cycle de détachement de poche peut se décrire de la manière suivante : 1) le jet rentrant provoquerait un vortex convecté à l'aval par l'écoulement extérieur jusqu'au collapse, 2) le collapse de ce vortex, devenu tourbillon en "fer à cheval ", conduit alors à une surpression qui provoque la remontée de liquide sous la poche formant ainsi le jet rentrant (comme le suggère le modèle instationnaire) (DELANNOY, KuENY, 1990).

La durée d'un cycle peut alors se décomposer en 2 temps successifs: d'une part, le temps de développement du vortex et de sa convection vers l'aval jusqu'au collapse sur une distance $\left(\mathrm{L}_{\text {jet }}+\Delta \mathrm{X}_{\text {coll }}\right)$ à la vitesse Vvor., et d'autre part, le temps de remontée du jet rentrant sous la poche sur une longueur, $\mathrm{L}_{\mathrm{jet}}$ :

$$
\Delta \mathrm{t}=\left(\mathrm{L}_{\mathrm{jet}}+\Delta \mathrm{X}_{\text {collapse }}\right) / \mathrm{V}_{\mathrm{vor}}+\mathrm{L}_{\mathrm{jet}} / \mathrm{V}_{\mathrm{jet}} .
$$

Le jet rentrant a pu être mis en évidence à l'aide de colorant injecté par les prises de pressions au niveau de la fermeture de la poche. La longueur de remontée du jet, Ljet, a pu être déterminée approximativement selon les 2 régimes de fonctionnement, périodique et non périodique :

* dans le cas de détachements périodiques (qui se produit pour de petites longueurs de poches à incidence nominale), le jet rentrant remonte jusqu'au bord d'attaque : la distance alors parcourue par le jet rentrant sous la poche, Ljet, est équivalente à la longueur de la cavité, Lcav. La fréquence, f, des détachements de structures diphasiques, mesurée par l'analyse spectrale des signaux de pression synchronisés à la visualisation, est inversement proportionnelle à la longueur moyenne de la cavité, Lcav, pour une vitesse amont donnée, Vref; ainsi, le nombre de Strouhal, St, est constant :

$$
\mathrm{St}=(\text { f.Lcav) } / \mathrm{Vref}=0.28 \pm 0.03 \text { (cf. fig. } 5 \text { ) }
$$

Dans ce cas, l'expression de la période du détachement est :

$$
\Delta \mathrm{t}=\mathrm{L}_{\text {cav }} / \text { St.Vref. }
$$

En égalant cette expression à l'équation (1) pour laquelle $\mathrm{Ljet}=\mathrm{Lcav}, \Delta \mathrm{X}_{\text {coll. }}$ doit être proportionnel à Lcav si l'on suppose que les vitesses moyennées Vvor et Vjet sont proportionnelles à Vref.

* Dans le cas de détachements non périodiques (il s'agit de fonctionnement avec de longues poches ou pour une incidence plus faible), la longueur de remontée du jet n'est plus constante, $\mathrm{L}_{\mathrm{jet}}$ est alors fluctuante, ce qui conduit à un $\Delta t$ variable (eq. (1)). En effet, dans ce cas, l'analyse spectrale des signaux de pression ne présente pas de fréquences caractéristiques [JouSSELLIN et al., 1991].

\section{Veine «Venturi»}

Bien que les formes de poches moyennes et les pressions moyennes mesurées dans la veine "Venturi " soient similaires à celles obtenues sur la veine " profil » [KUENY et al., 1991], les comportements instationnaires diffèrent: les structures en "fer à cheval " sont visibles mais leur lâcher est non périodique [Joussellin et al., 1991].

Les spectres de pressions se rapprochent alors de celui d'un bruit blanc pour une poche courte, ils contiennent une fréquence dominante de plus en plus basse quand la longueur de la cavité augmente (fig. 6).

Les principales différences entre les 2 modèles bidimensionnels qui peuvent influer sur le comportement instationnaire des cavités, sont le confinement (cf. $t a b l .1$ ) et les couches limites (fig. 3).

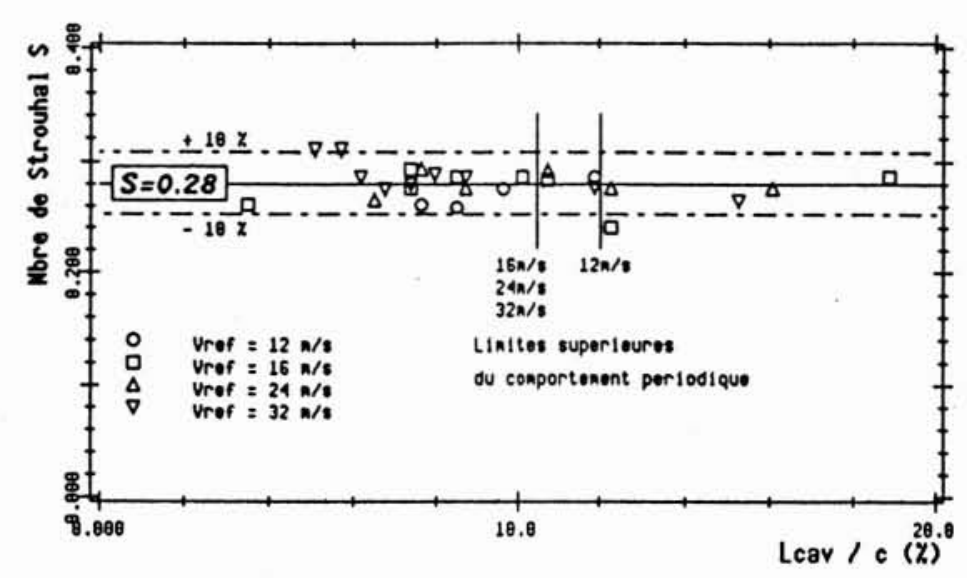

5. Nombre de Strouhal: $S t=f . L_{\text {cav }} / V_{\text {ref, }}$ en fonction de la longueur de poches, $L_{c a v}$.

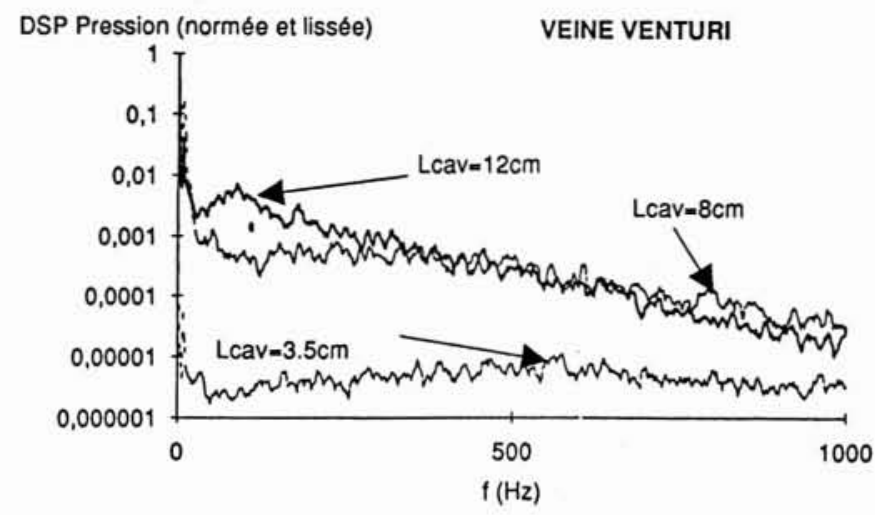

6. Spectres de pression pariétale pour 3 longueurs de poches $L_{\text {cav }}$. 


\section{Inuducteur à l'eau}

L'objectif est de caractériser les instabilités sur le modèle tournant qui se manifestent entre autre par d'importants efforts fluctuants sur la ligne d'arbre, en régime cavitant. Les études précédentes ont montré la complexité de l'écoulement dans ce type de machines soumis aux phénomènes de cavitation [ACOSTA, 1992], avec une forte dépendance de comportement en fonction du nombre de pales (cf. inducteur 3 pales [KАMiJo et al., 1977]).

L'étude est réalisée à $\mathrm{QN}$ pour 3 vitesses de rotation ( $3000 \mathrm{tr} / \mathrm{mn}, 5500 \mathrm{tr} / \mathrm{mn}, 7500 \mathrm{tr} / \mathrm{mn}$ ). Pour chaque régime, 5 niveaux de pression amont ont permis d'étudier le développement de la cavitation. En considérant des valeurs identiques du nombre de cavitation, on retrouve les mêmes figures de cavitation pour les 3 vitesses.

La figure 7 montre les figures de cavitation observées en fonction du nombre de cavitation, $\sigma$, et les spectres de pression associés :

* pour des $\sigma$ élevés (cas 1 et 2), il s'agit de points de fonctionnement stables du type: 1 petite poche par pale (fréq. caractéristique : $f=4 \cdot f_{\text {rot }}$ ) ou encore, pour un $\sigma$ plus faible, 1 courte $/ 1$ longue $/ 1$ courte... $\left(f=2 \cdot f_{\text {rot }}\right)$;

** pour des $\sigma$ plus petits, une plage d'instabilités apparaît, liée à l'apparition d'une poche courte qui tourne à une vitesse supersynchrone (cas 3); la fréquence associée, $f>f_{\text {ror }}$, diminue avec $\sigma$, puis se synchronise à la rotation de l'arbre $\left(f=f_{\text {rot }}\right)$. Cette dernière configuration « 1 poche courte/3 longues" (cas 4) est entrecoupée de séquences comportant 4 longues poches. Ces régimes dissymétriques conduisent à des efforts radiaux sur l'arbre de l'inducteur très importants [GoIRAND et al., 1992];

*** pour de faibles $\sigma$, les pales sont recouvertes par les poches jusqu'au niveau du col (section minimum interaube); une longueur des poches supérieure à ce seuil conduit à une perte de performances de la machine. Cette configuration avec une grande poche par pale (cas 4) est stable $\left(f=4 \cdot f_{\text {rot }}\right)$.

L'étude plus détaillée des configurations instables est entreprise à partir des enregistrements des signaux de pression, d'où est extraite une séquence temporelle caractéristique du phénomène d'instabilités (fig. 8) de l'essai 23 $(\sigma=0,04, \quad 7500 \mathrm{tr} / \mathrm{mn}, \quad f o=125 \mathrm{~Hz})$. La fréquence prépondérante est dans ce cas, $f=143 \mathrm{~Hz}(f \mid f o=1.14$, fig. 7 (cas 3)). La séquence visuelle se décompose en 7 phases (une par pale: $\Delta t=1 / 4$.tour): 1 poche courte $/ 3$ longues $/ 1$ courte $/ 2$ longues.

A partir de la visualisation par films rapides on a pu en déduire une vue globale de l'inducteur en suivant l'évolution successive de la cavitation sur les 4 pales (alors que la prise de vue est limitée à $1 / 3$ tour). Cette séquence montre que les longues poches sont associées à de la cavitation d'entrefer, sauf à l'amont de la petite poche qui est une zone de surpression. La petite poche remonte de pale en pale tous les $7 / 4$ de tour.

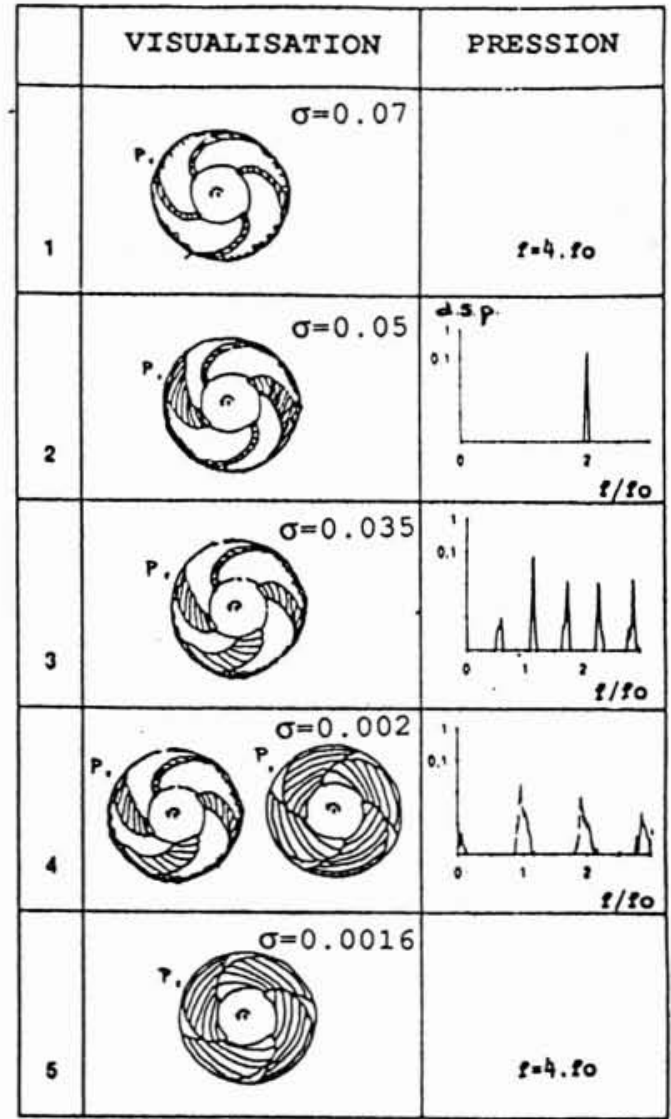

7. Figures de cavitation et spectres de pression en fonction du paramètre de cavitation, $\sigma(P$ : position du capteur de pression $)$.

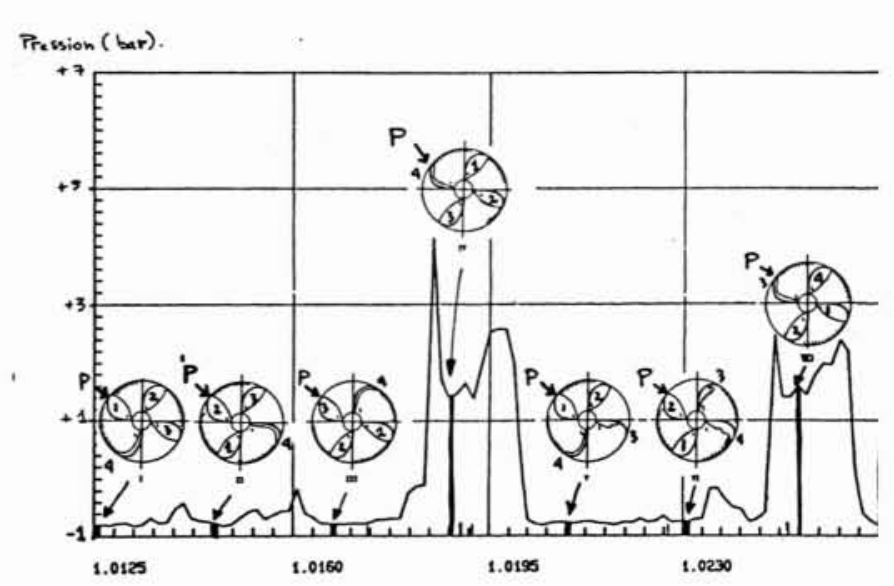

8. Synchronisation du signal temporel de pression et de la visualisation par films rapides (étendue à 4 pales): séquence de $14 \mathrm{~ms}$ (essai 23). 


\section{Conclusion}

Ces 3 expériences ont montré différents comportements instationnaires: dans les modèles bidimensionnels, des lâchers de structures de vapeur ont été observés (périodique pour la veine " profil ", non périodique pour la veine «Venturi » (différence de confinement ou de couches limites)). Dans l'inducteur, une grille d'analyse des instabilités a été développée dans laquelle apparaissent des configurations cavitantes dissymétriques, qui pourraient être à l'origine d'efforts importants sur l'arbre.

Tous les régimes instables montrent que la fréquence associée aux phénomènes stationnaires diminue quand la longueur des poches de cavitation augmente (ou $\sigma$ diminue).

\section{Bibliographie}

Acosta A.J. - «Flow in inducer pumps, an aperçu ", 4th Int. Symp. on Transport phenomena and dynamics of rotating machinery, Honolulu, Hawai, April 5-8 1992.

Delannoy Y., Kueny J.L. - "Two phase flow modelling for unsteady cavitation ", ASME cavitation \& multiphase flow forum, Toronto, juin 1990.

Goirand B., Mertz A.L., Joussellin F., Rebattet C. « Experimental investigations of radial loads induced by partial cavitation with the $\mathrm{LH}_{2}$ VULCAIN inducer ", $3 r d$ international conference on Cavitation, Cambridge, UK, 9-11 Dec. 1992.

Joussellin F., Delannoy Y., Sauvage-Boutar E., Goirand B. (1991). - « Experimental investigations on unsteady attached cavities", ASME 1991 Cavitation Symposium, Portland, Oregon, June 1991.

Kamijo K., Shimura T., Watanabe M. - «An experimental investigation of cavitating inducer instability ", $A S M E$ paper 77-WA/FE-14, 1977.

Kueny J.L., Desclaux J. (1989), - "Theoretical analysis of cavitation in rocket engine inducers ", ASME Pumping Machinery Symposium, San Diego, USA, Dec. 1989.

Kueny J.L., Reboud J.L., Desclaux J. (1991). - « Analysis of partial cavitation by image processing \& numerical prediction ", ASME 1991 Cavitation Symposium, Portland, Oregon, USA, June 1991. 


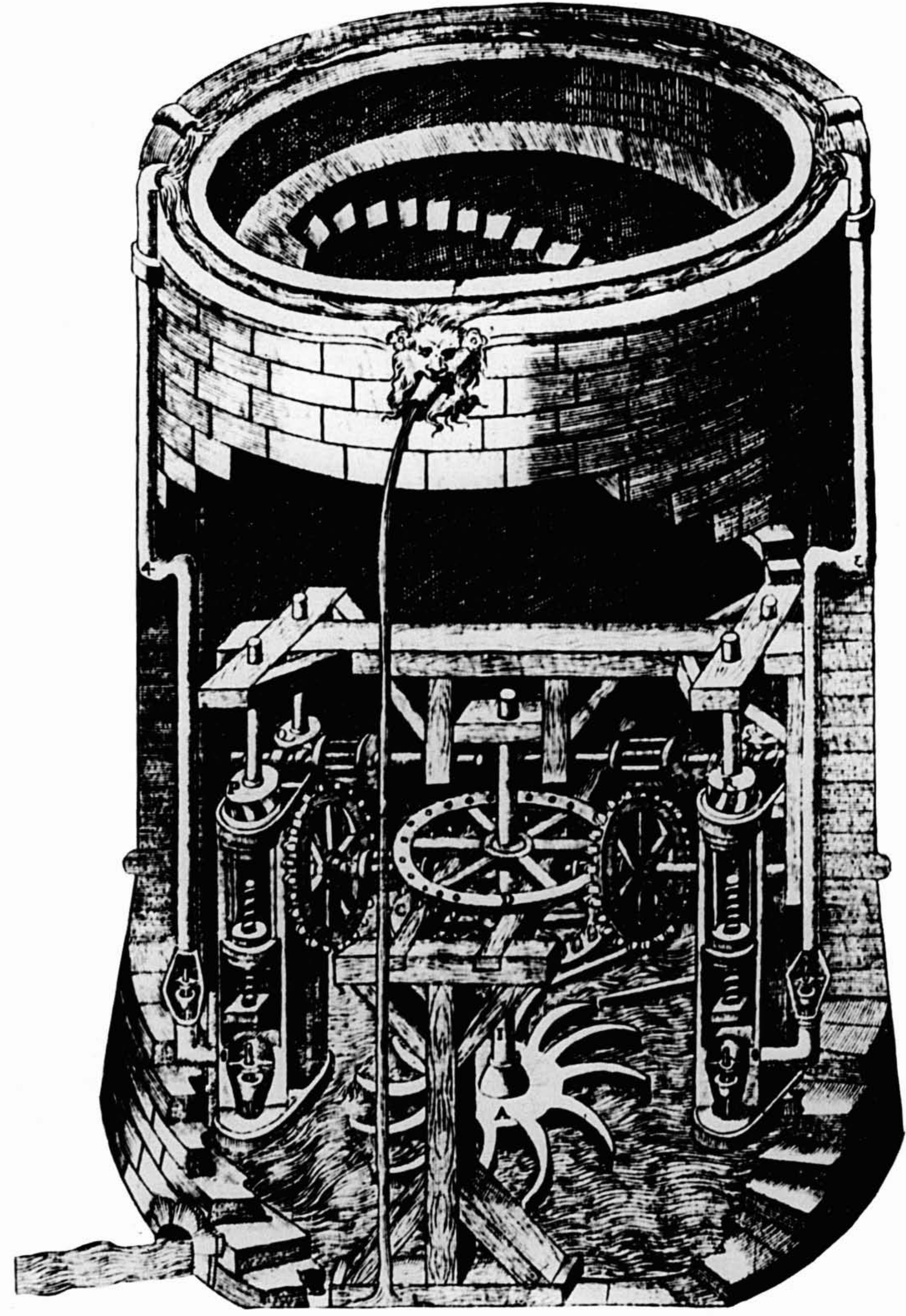

